\title{
Propiedades psicométricas de la Escala de Desviación de la Norma en jóvenes
}

\section{Psychometric properties of the Normative Deviance Scale in youths}

\author{
Sebastián Jesús Garrido ${ }^{\mathrm{a}, \mathrm{b}, ~ *}$, Karin Arbachª b , Marcos Cupania ${ }^{\mathrm{a}, \mathrm{b}}$, Fernanda Belén Ghío ${ }^{\mathrm{a}, \mathrm{b}}$, \\ Ana Estefanía Azpilcueta ${ }^{\mathrm{a}, \mathrm{b}}$, Valeria Estefanía Morán ${ }^{\mathrm{a}, \mathrm{b}}$ \\ aFacultad de Psicología, Universidad Nacional de Córdoba, Argentina \\ ${ }^{b}$ Instituto de Investigaciones Psicológicas, Unidad Ejecutora CONICET, Argentina
}

\section{Resumen}

Antecedente: La conducta antisocial refiere al conjunto de actos que violan las normas establecidas para la convivencia de una sociedad. Entre las medidas de autoinforme para valorar esta conducta se encuentra la Escala de Desviación de la Norma (EDN). Objetivo: Considerando que en el contexto argentino no se encontraron estudios sobre las propiedades psicométricas de la escala, el objetivo de este estudio fue analizar sus propiedades psicométricas en una muestra de jóvenes argentinos. Método: Para este fin, se conformó una muestra de 794 participantes con edades comprendidas entre los 18 y 25 años. Se realizó un análisis factorial confirmatorio, se analizó el índice de confiabilidad compuesta y se estudió la validez convergente, discriminante y concurrente (utilizando una escala de autocontrol). Además, se analizó la invarianza del modelo de medida. Resultados: Entre los principales resultados se confirmó la estructura factorial original de la escala sin equivalencia factorial entre los grupos evaluados considerando el sexo, y se encontraron adecuadas propiedades psicométricas para la mayoría de las dimensiones que componen el instrumento. Discusión: Se discuten los hallazgos, limitaciones y futuras líneas de investigación.

Palabras clave: conducta antisocial, Escala de Desviación de la Norma, jóvenes, autocontrol.

\section{Para citar este artículo:}

Garrido, S. J., Arbach, K., Cupani, M., Ghío, F. B., Azpilcueta, A. E., \& Desviación de la Norma en jóvenes. Liberabit, 25(2), 233-249. https:// doi.org/10.24265/liberabit.2019.v25n2.07 Morán, V. E. (2019). Propiedades psicométricas de la Escala de

\begin{abstract}
Background: Antisocial behavior refers to a set of acts that violate the rules established for the coexistence of society. One of the self-report measures to assess this type of behavior is the normative deviance scale (NDS). Objective: Considering that in the Argentine context no studies on the psychometric properties of the scale were found, the present study aimed to analyze the psychometric properties of the scale in a sample of Argentine youths. Method: To this end, a sample of 794 participants aged between 18 and 25 years was collected. A confirmatory factor analysis was performed; the composite reliability was calculated; and the convergent, discriminant and concurrent validity was assessed (using a self-control scale). In addition, the invariance of the factorial structure of the scale was analyzed. Results: The results confirmed the original factorial structure of the scale with no factorial equivalence across the assessed groups regarding the sex. Also, the results showed adequate psychometric properties for most dimensions which make up the instrument. Conclusions: Findings, limitations and further lines of research are discussed.
\end{abstract}

Keywords: antisocial behavior; normative deviance scale, youths, self-control.

Este es un artículo Open Access bajo la licencia Creative Commons Atribución-NoComercial-CompartirIgual 4.0

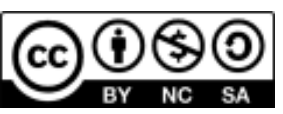

Universidad de San Martín de Porres, Lima - Perú

* sebajgarrido@gmail.com 


\section{Introducción}

La conducta antisocial puede definirse como el conjunto de actos que violan las normas establecidas para la convivencia de una sociedad (Gaik, Abdullah, Elias, \& Uli, 2010; Moffitt, 1993). Considerando las consecuencias que genera a nivel individual y social, existe un amplio interés científico, académico y de las instituciones públicas para conocer el inicio, desarrollo, prevalencia, desistimiento de la conducta antisocial, como así también de los factores de riesgo explicativos de su aparición (Butchart \& Mikton, 2014; Moffitt, 1993).

De acuerdo con los datos estadísticos de la Organización Mundial de Salud (OMS), el 43\% del total de los homicidios que ocurren a nivel internacional involucran a individuos 3/4como víctima o victimario $3 / 4$ entre los 10 y 29 años, de los cuales el 83\% son de sexo masculino (World Health Organization, 2015). Este nivel de prevalencia convierte al homicidio en la cuarta causa de defunción en personas de esta franja etaria. Asimismo, se estima que por cada joven asesinado entre 20 y 40 sufren lesiones que requieren de tratamiento hospitalario (World Health Organization, 2015).

En Argentina en el año 2017 se registró a nivel nacional que un $96 \%$ de quienes se encontraban privados de su libertad fueron de sexo masculino y que el 60\% tenían menos de 35 años (Ministerio de Justicia y Derechos Humanos, 2017a). Por su parte, en la provincia de Córdoba, en el mismo año se reportó que el 95\% de la población en las cárceles eran hombres y el $58 \%$ se localizó en la franja etaria entre los 18 y los 35 años (Ministerio de Justicia y Derechos Humanos, 2017b).

Con respecto a las diferencias en el comportamiento antisocial según el sexo, estudios de investigación previos confirmaron que, salvo ciertas excepciones, los hombres se involucran con mayor frecuencia en comportamientos antisociales o delictivos (Eitle, Niedrist, \& Eitle, 2014). Entre las diferencias encontradas en la literatura se identificó que los hombres beben con mayor frecuencia y consumen cantidades más elevadas de alcohol que las mujeres (Chaiyasong et al., 2018). Además, los hombres presentaron mayor prevalencia que las mujeres en comportamientos de agresión directa (e.g., agresión física) (Card, Stucky, Sawalani, \& Little, 2008), en comportamiento vandálico (i.e., grafftis) (Nordmarker, Hjärthag, Perrin-Wallqvist, \& Archer, 2016) y de hurto en las tiendas (Hoertel, Dubertret, Schuster, \& Le Strat, 2012).

Entre los rasgos de la personalidad que se han vinculado con el comportamiento antisocial se encuentra el autocontrol. La teoría general del delito propone que altos niveles en el rasgo latente autocontrol, se asocian con la evitación de actos y comportamientos inmediatos o momentáneos, cuyos costos y consecuencias excedieran sus beneficios a largo plazo (Gottfredson \& Hirschi, 1990). Mientras que en aquellos individuos con bajos niveles de autocontrol sería superior la probabilidad de cometer cualquier acto antisocial o comportamiento análogo al delito (Gottfredson \& Hirschi, 1990). De esta manera, siguiendo con la propuesta teórica de la teoría general del delito, se podrían identificar, por una parte, aquellas conductas asociadas a elevados niveles de autocontrol como, por ejemplo, el éxito académico, hábitos alimenticios saludables y relaciones interpersonales satisfactorias (Tangney, Baumeister, \& Boone, 2004). Y por otra parte, se podrían agrupar las conductas vinculadas con bajos niveles de autocontrol como el robo (Chui \& Chan, 2013), el consumo excesivo de alcohol (Walters, Simons, \& Simons, 2018), el uso de drogas (Baron, 2003) y la violencia escolar (Tillyer, Wilcox, \& Fissel, 2018).

Con respecto a la valoración de la conducta antisocial y delictiva, estas se realizan usualmente a partir de registros oficiales e instrumentos de autoinforme. Si bien suele reconocerse que los registros oficiales presentan mayor fiabilidad (por basarse en sentencias judiciales), en el campo de la psicología las medidas de autoinforme son utilizadas con mayor frecuencia. Esto se debe a que permiten evaluar una 
variedad más amplia de conductas en contra de la norma y a que consideran conductas de menor gravedad y seriedad delictiva que aquellas que contemplan los registros oficiales (Thornberry \& Krohn, 2000; Webb, Katz, \& Decker, 2006).

Entre las medidas de autoinforme que consideran como fundamento teórico los postulados de la teoría general del delito sobre la medición de la delincuencia y la desviación social (Gottfredson \& Hirschi, 1990), se encuentra la Escala de Desviación de la Norma (EDN; Vazsonyi, Pickering, Junger, \& Hessing, 2001). Esta escala permite valorar diferentes tipos de conductas antinormativas (desviación social) o antisociales, independientemente de las definiciones legales que pueden existir de las conductas delictivas. Esta característica de la escala permite su administración en diferentes países y culturas, ya que sus ítems indagan sobre conductas de menor gravedad que las delictivas, lo que posibilita evaluar la desviación social en personas de distintas edades, distintos géneros y diferentes sociedades, incluso en aquellas que presentan menores niveles de delincuencia (Vazsonyi et al., 2001).

De acuerdo con Vazsonyi et al. (2001), la EDN se compone por siete factores. El factor Vandalismo, que permite evaluar daño intencional de bienes que no pertenecen al individuo; Consumo de alcohol, para valorar el consumo de alcohol cuando se encuentra prohibido por la ley (o el consumo abusivo de alcohol solo por diversión); Consumo de drogas, para evaluar el consumo de drogas legales e ilegales (e.g., tabaco, marihuana, éxtasis); Robo, para valorar la participación del individuos en actos de sustracción de bienes ajenos; Agresión física, para evaluar la amenaza o la participación del individuo en conductas que involucran violencia física; Mala conducta escolar, para evaluar las sanciones que recibió un individuo en un entorno educativo o aquel comportamiento escolar que podría recibir sanciones escolares; y el factor Desviación general, que evalúa el comportamiento antisocial de carácter más amplio. Asimismo, los autores han propuesto la posibilidad de obtener una medida general de comportamiento de desviación social mediante la sumatoria de todos los ítems que componen la escala (Vazsonyi et al., 2001).

Con respecto a las propiedades psicométricas de la escala, estudios previos informaron la validez y confiabilidad de la versión inicial de siete factores a través de muestras de diferentes culturas (Vazsonyi et al., 2001; Vazsonyi, Wittekind, Belliston, \& Loh, 2004). Por ejemplo, en relación con la validez de la escala considerando la asociación de la variable desviación social con constructos vinculados como impulsividad, búsqueda de sensaciones y temperamento volátil se encontraron correlaciones positivas entre estas variables con valores de $r$ entre .14 y .40 (Vazsonyi et al., 2001). Por su parte, la confiabilidad de la escala valorada mediante el índice de confiabilidad de Cronbach varió para las siete dimensiones entre .77 y .90 en población general (Coffey, Cox, \& Kopkin, 2018), entre .65 y .87 en muestras compuestas por adolescentes en conflicto con la ley penal (Bobbio, Arbach, \& Vazsonyi, 2019) y entre .77 y .86 en muestras de adolescentes sin conflicto con la ley penal (Torrente \& Vazsonyi, 2012).

Cabe mencionar, que pese al uso frecuente de la EDN en diversos contextos culturales (e.g., Alemania, China, España, Estados Unidos, Holanda, Hungría, Japón, Turquía), los estudios que la utilizaron suelen reportar los índices de confiabilidad que presenta cada una de las dimensiones de la escala (en la muestra utilizada) y remitirse a los estudios de validez presentados en el manuscrito original, sin manifestar como objetivo de investigación la evaluación de las propiedades psicométricas de la EDN. Considerando lo antes mencionado -que en el contexto argentino no se cuenta con una prueba con las características de evaluación de la EDN y que tanto a nivel nacional como local se reportan elevadas tasas de conductas antisociales durante la juventud-, el objetivo de investigación de este estudio es analizar las propiedades psicométricas de la escala en una muestra de jóvenes argentinos para determinar si es un 
instrumento adecuado para ser aplicado en esta población.

\section{Método}

\section{Diseño de investigación}

Siguiendo la clasificación de Montero y León (2007) y considerando que el objetivo del presente estudio es el análisis de las propiedades psicométricas de la EDN, el diseño de investigación es instrumental.

\section{Participantes}

La muestra estuvo compuesta por 794 jóvenes de ambos sexos, 625 participantes mujeres (79\%) y 169 varones (21\%). Las edades de los participantes se encontraron comprendidas entre los 18 y 25 años ( $M$ = 21.64; $D E=2.11)$. Los participantes fueron estudiantes de las facultades dependientes de la Universidad Nacional de Córdoba (Argentina): Facultad de Arte (3\%); Facultad de Ciencias Agropecuarias (.4\%); Facultad de Arquitectura, Urbanismo y Diseño (2.5\%); Facultad de Derecho y Ciencias Sociales (11.3\%); Facultad de Ciencias Económicas (5.8\%); Facultad de Matemática, Astronomía y Física (3.3\%); Facultad de Ciencias Exactas, Físicas y Naturales (14\%); Facultad de Ciencias Médicas (14.6\%); Facultad de Odontología (2.4\%); Facultad de Filosofía y Humanidades (3.8\%); Facultad de Lengua (0.9\%); Facultad de Psicología (15\%); Facultad de Ciencias Químicas (2.8\%); otra (.9\%) y casos perdidos (19.3\%).

\section{Instrumentos}

Escala de Desviación de la Norma (Normative Deviance Scale; Vazsonyi et al., 2001). La EDN está conformada por 55 ítems que se agrupan en 7 factores: Vandalismo, Consumo de alcohol, Consumo de drogas, Mala conducta escolar, Desviación general, Robo y Agresión física. En la consigna de la escala se les solicita a los participantes que indiquen cuál es la frecuencia con la que realizaron las afirmaciones que se presentan en cada ítem, utilizando un formato de respuesta tipo Likert con 5 opciones de respuesta que van desde 1 (nunca) hasta 5 (6 veces o más). Las propiedades psicométricas de la escala reportadas por los autores indican que los estudios de consistencia interna reportaron valores alfa de Cronbach que oscilaron entre .76 y .89. Para la traducción de los ítems de la escala tres expertos de la lengua inglesa realizaron, de manera independiente, una traducción directa de la versión original del inventario al español. Las tres versiones fueron comparadas y sometidas a discusión considerando diferencias lingüísticas y culturales entre la población de origen y la población de destino.

Autocontrol. Para evaluar este constructo se utilizó la Escala Breve de Autocontrol (BSCS, por sus siglas en inglés; Tangney et al., 2004) en su versión adaptada por Garrido, Cupani y Arbach (2017). Esta escala está compuesta por 13 ítems que poseen un formato de respuesta tipo Likert con 5 opciones de respuesta que van desde 1 (para nada de acuerdo) hasta 5 (totalmente de acuerdo). La confiabilidad evaluada por los autores mediante el coeficiente de confiabilidad omega fue igual a .81 (Garrido et al., 2018). En relación con la validez, Tangney et al. (2004) reportaron que las personas con puntajes más elevados en la escala BSCS presentan un mejor ajuste psicológico, mejores habilidades interpersonales y menos problemas de comportamiento como los atracones de comida o el consumo excesivo de alcohol.

\section{Procedimiento}

La difusión del estudio se realizó mediante correo electrónico o grupos de Facebook universitarios. La invitación enviada contenía una descripción del estudio y un enlace que redirigía automáticamente a la plataforma virtual LimeSurvey que ofrece el servidor de encuestas de la Universidad Nacional de Córdoba. Una vez dentro del servidor, el participante se encontraba con un texto en el cual se presentaba la finalidad de la encuesta, su carácter anónimo y los datos de contacto del responsable del estudio. 
Asimismo, considerando las recomendaciones de la legislación de la República Argentina (Ley N 25.326, 2000) y de la American Psychological Association (APA, 2016), se les informó a los jóvenes sobre la confidencialidad de sus respuestas, el carácter voluntario de su participación y su derecho a retirarse en cualquier instancia del estudio, sin que esto conlleve algún perjuicio para su persona. Además, en esta primera página de la encuesta si el joven presionaba el botón con la leyenda «aceptar» estaba otorgando su consentimiento para formar parte del estudio. Con respecto al tiempo requerido para completar las escalas el promedio de tiempo calculado fue de 25 minutos aproximadamente.

\section{Análisis de datos}

Se utilizó el software SPSS para Windows versión 19.0 con el fin de conocer la prevalencia de la conducta antisocial y así preparar los datos para los análisis estadísticos de la estructura interna de la escala. Para conocer la presencia del comportamiento antisocial en la muestra se recodificaron las respuestas en dos categorías: al menos una vez llevaron a cabo el comportamiento antisocial (opciones de respuesta 2 a 5) y nunca lo hicieron. Luego de analizar la prevalencia, se evaluaron las distribuciones de las respuestas de cada uno de los ítems (con su escala original de 5 puntos de respuesta) considerando la asimetría y la curtosis. Como criterio para evaluar los índices de asimetría y curtosis se interpretó como excelentes valores entre \pm 1 y adecuados valores inferiores a \pm 2 (George \& Mallery, 2011).

Para evaluar la estructura factorial de la EDN se realizó un análisis factorial confirmatorio en el software Mplus 6.1 (Muthén \& Muthén, 2011). Considerando que el instrumento posee una escala de respuesta tipo Likert, se estimó el modelo utilizando correlaciones policóricas y como método de estimación la técnica de robustos mínimos cuadrados ponderados (WLS, por sus siglas en ingles). Para evaluar el ajuste del modelo se utilizaron los siguientes índices y sus respectivas interpretaciones (Yu \& Muthén, 2002): chi-cuadrado $\left(\chi^{2}=>\right.$.05); índice de ajuste comparativo (CFI) e índice de Tucker-Lewis (TLI) (> .90); raíz cuadrada media del error de aproximación (RMSEA < .05) y media cuadrática residual ponderada (WRMR $<1$ ).

Para realizar las modificaciones al modelo de medida se consideraron los valores del índice de modificación superiores a lo recomendado en la literatura (3.84), los cuales indicarían una reducción estadísticamente significativa en el coeficiente chicuadrado. Conjuntamente se interpretaron los valores estandarizados del cambio esperado en el parámetro (ZEPC, por sus siglas en inglés) siempre y cuando hayan sido interpretables, significativos y mejoren el ajuste del modelo (Brown, 2006; Gunzler \& Morris, 2015; Whittaker, 2012). Además, se evaluaron los pesos factoriales de los ítems, considerándose como criterio de exclusión las cargas factoriales estandarizadas $(\lambda)$ inferiores a .40 (Nunnally, 1978).

Para el estudio de consistencia interna se calculó el coeficiente de confiabilidad compuesta considerando adecuados valores superiores a .70 (Nunnally, 1978). Con respecto a la validez basada en la asociación con otras variables se evaluó, por una parte, la validez convergente mediante la AVE (varianza media extraída) que proporciona información sobre la cantidad de varianza explicada del constructo por cada uno de sus indicadores. Para este índice se considera adecuado un valor superior a .40 (Etchezahar, PradoGascó, Jaume, \& Brussino, 2014). Por otra parte, se analizó la validez discriminante y se consideraron los índices MSV (máxima varianza compartida al cuadrado) y la ASV (varianza media al cuadrado compartida), cuyos valores deben ser menores que la AVE de cada uno de los factores.

Con la finalidad de obtener mayor evidencia de validez de la escala, se procedió a evaluar la evidencia de validez relacionada al criterio de tipo concurrente para lo cual se realizó un análisis de correlación bivariado entre las siete dimensiones de la EDN y la variable Autocontrol. Considerando lo propuesto por la teoría general del delito se esperó encontrar 
relaciones negativas entre estas variables (Gottfredson \& Hirschi, 1990). Para interpretar el coeficiente de correlación de Pearson se consideró como un nivel de asociación bajo los valores de correlación de .10; medio, los valores de correlación .30; y altos, los valores de correlación .50 (Cohen, 1988).

Por último, se evaluó la invarianza factorial considerando el sexo de los participantes (Byrne, 2008). Con el modelo de medida final, se realizó la evaluación de la invarianza configural para determinar si el número de los factores son equivalentes en ambos grupos, de la invarianza métrica para conocer si los patrones de saturación factorial de los ítems en cada factor son iguales y de la invarianza escalar para evaluar la igualdad entre las intersecciones de los ítems entre los grupos (Byrne, 2008; Dimitrov, 2010). Cabe mencionar que, para interpretar los resultados del análisis de invarianza, es usual comparar los modelos anidados mediante la razón de máxima verosimilitud o test de la diferencia del estadístico chi-cuadrado (Hair, Black, Babin, \& Anderson, 2010). Sin embargo, esta prueba tiene una alta sensibilidad ante el tamaño de la muestra y frente al incumplimiento del supuesto de normalidad de los datos (Hair et al., 2010; Chen, 2007). Por este motivo, en este estudio se consideró el cumplimiento del supuesto de invarianza factorial si entre los modelos analizados las diferencias entre el CFI no fueran superiores a $.01(\Delta \mathrm{CFI} \leq .01)$, entre el TLI no fueran superiores a $.01(\Delta \mathrm{TLI} \leq .01)$ y entre los RMSEA no fueran superiores a $.015(\Delta$ RMSEA $\leq .015)$ (Chen, 2007; Cheung \& Rensvold, 2002).

\section{Resultados}

\section{Resultados descriptivos}

Como resultado de los análisis descriptivos se obtuvo que, de la totalidad de la muestra, más del $90 \%$ al menos una vez consumió alcohol, presentó una conducta inadecuada en la escuela o cometió algún hecho de desviación general. Más del 80\% consumió drogas consideradas ilegales, más del 65\% cometió algún acto considerado vandálico y menos del 40\% cometió actos de robo o lesiones. Cuando se consideraron los porcentajes dentro de cada grupo, se encontró que el porcentaje de varones que habían cometido al menos una vez un acto antisocial fue superior al de aquellos que nunca lo habían cometido. Por su parte, en el grupo de las mujeres, a excepción de las dimensiones agresión y robo, el comportamiento fue similar al de los hombres (ver Tabla 1).

\section{Análisis de ítems}

Se analizaron los índices de asimetría y curtosis para cada uno de los ítems. El análisis de asimetría mostró que 13 ítems de la escala presentaron valores comprendidos entre +1 y $-1,11$ ítems presentaron índices inferiores a $2 \mathrm{y}$ el resto de los ítems presentaron valores superiores a 2 . Con respecto a la curtosis, ocho ítems presentaron valores entre -1 y $+1,11$ ítems presentaron valores inferiores a 2 y el resto de los ítems presentaron valores superiores a 2 .

\section{Análisis inicial de la estructura factorial de la EDN}

Los resultados del análisis factorial confirmatorio indicaron que el modelo de medición de siete factores no se ajusta adecuadamente a los datos: $\chi^{2}=8629.915$ $(p<.01)$, CFI .61, TLI .59, RMSEA .082 IC 90\% [0.081 - 0.084], WRMR 3.23. Considerando estos resultados se procedió al análisis de consistencia interna de este modelo de medición y se encontró que el índice de confiabilidad compuesta varió en un rango de .70 (Desviación general) hasta .93 (Consumo de droga). Sin embargo, al evaluar la validez convergente y discriminante se encontró que en la mayoría de las dimensiones evaluadas los índices MSV y ASV fueron superiores a los valores obtenidos para la AVE. Aunque cabe mencionar que solo en las dimensiones Consumo de alcohol y Consumo de drogas el valor de ASV cumplió con el criterio ASV < AVE. Luego de obtener estos resultados sobre la estructura factorial, la confiabilidad y validez del modelo de medida original, se procedió a examinar los pesos factoriales y los índices de modificación para mejorar el ajuste del modelo estadístico. En consecuencia, se eliminaron 
Tabla 1

Análisis de frecuencia (\%) de los participantes por subescala según la realización de al menos una vez o si nunca llevaron a cabo el comportamiento antisocial

\begin{tabular}{lccc}
\hline & $\begin{array}{c}\text { Total } \\
\text { N }=794 \\
\%\end{array}$ & $\begin{array}{c}\text { Mujeres } \\
n=625 \\
\%\end{array}$ & $\begin{array}{c}\text { Varones } \\
n=169 \\
\%\end{array}$ \\
$\begin{array}{lccc}\text { Vandalismo } \\
\quad \text { Nunca }\end{array}$ & 31.7 & 35 & 19.5 \\
$\quad$ Al menos 1 vez & 68.3 & 65 & 80.5 \\
Consumo de alcohol & & & \\
$\quad$ Nunca & 5.2 & 5.9 & 2.4 \\
$\quad$ Al menos 1 vez & 94.8 & 94.1 & 97.6 \\
Consumo de drogas & & & \\
$\quad$ Nunca & 16.6 & 18.1 & 11.2 \\
$\quad$ Al menos 1 vez & 83.4 & 81.9 & 88.8 \\
Mala conducta escolar & & & \\
$\quad$ Nunca & 4.7 & 5.1 & 3 \\
$\quad$ Al menos 1 vez & 95.3 & 94.9 & 97 \\
Desviación general & & & \\
$\quad$ Nunca & & 7.8 & 5.3 \\
Al menos 1 vez & 92.7 & 92.2 & 94.7 \\
Robo & & & \\
$\quad$ Nunca & & 63.8 & 49.1 \\
Al menos 1 vez & 39.3 & 36.2 & 50.9 \\
Agresión física & & & \\
$\quad$ Nunca & 60.3 & 65.8 & 40.2 \\
Al menos 1 vez & 39.7 & 34.2 & 59.8 \\
\hline
\end{tabular}

los ítems: 5, 8 (Vandalismo), 24 (Consumo de drogas), 27 (Mala conducta escolar), 33, 34, 36 (Desviación general), 46, 48, 49 (Robo), 53 y 54 (Agresión física). Además, el índice de modificación de ajuste sugirió realizar la covarianza entre los errores de los ítems 9 y 10, y de los ítems 16 y 17.

\section{Análisis de la estructura factorial final de la EDN}

Como resultado de las modificaciones realizadas al modelo de medida original, los índices de ajuste del modelo se ubicaron dentro de valores considerados adecuados: $\chi^{2}=1632.795(p<.01)$, CFI $=.948$, TLI $=.944$, RMSEA $=.034$ IC 90\% [.031 - .036], WRMR $=1.29$. De este modo, el modelo de medición final quedó compuesto por la dimensión Vandalismo (seis ítems), Consumo de alcohol (siete ítems), Consumo de drogas (ocho ítems), Mala conducta escolar (seis ítems), Desviación general (ocho ítems), Robo (cuatro ítems) y Agresión (cuatro ítems) (ver Tabla 2). 


\section{Tabla 2}

Cargas factoriales de los ítems por dimensión, análisis de confiabilidad, análisis de validez discriminante, convergente y concurrente

\begin{tabular}{|c|c|c|c|c|c|c|}
\hline Subescalas & $\lambda$ & $\mathrm{CC}$ & AVE & MSV & ASV & A \\
\hline Vandalismo & & .76 & .35 & .14 & .08 & $-.28^{*}$ \\
\hline
\end{tabular}

1. ¿Rompiste botellas en la calle, en el patio del colegio o en otras zonas?

.66

2. ¿Has dañado o destruido intencionadamente cosas que pertenecían a tus padres 0 a otros miembros de tu familia (e.g., hermanos/as)?

3. ¿Has dañado o destruido intencionadamente cosas que pertenecían a la escuela 0 la universidad?

4. ¿Has dañado o destruido intencionadamente otros objetos (e.g., carteles, ventanas, buzones, cajeros) que no te pertenecían?

5. ¿Has dañado de alguna forma (e.g., con un cuchillo, trincheta o lapicera) los asientos del colectivo, del cine o de otro lugar público?

6. ¿Escribiste grafitis en el colectivo, en las paredes de la escuela o universidad, en las paredes de algún baño o en cualquier otro lugar público?

\section{Consumo de alcohol}

7. ¿Consumiste bebidas alcohólicas con alta graduación (e.g., tequila, whisky, vodka o ginebra) antes de los 18 años?

8. ¿Consumiste otras bebidas alcohólicas (e.g., cerveza o vino) antes de los 18 años?

9. ¿Te emborrachaste intencionadamente solo por diversión (a cualquier edad)?

10. ¿Te emborrachaste solo para ser aceptado como miembro de un grupo (a cualquier edad)?

11. ¿Mentiste sobre tu edad para comprar alcohol antes de cumplir los 18 años?

12. ¿Hiciste que tu hermano/a o amigo/a mayor comprara alcohol por vos?

13. ¿Compraste alcohol para tu hermano/a o para algún amigo/a menor de edad?

\section{Consumo de droga}

14. ¿Consumiste regularmente productos con tabaco (e.g., cigarrillos o pipa)?

15. ¿Consumiste drogas blandas como marihuana?

16. ¿Consumiste drogas duras como cocaína o éxtasis?

17. ¿Fuiste a la escuela o a la universidad bebido o drogado?

18. ¿Fuiste a trabajar bebido o drogado?

19. ¿Fuiste a un recital bebido o drogado?

20. ¿Fuiste a una discoteca o a una fiesta bebido o drogado?

21. ¿Fuiste a una discoteca o a una fiesta para emborracharte 0 drogarte?

\section{Mala conducta escolar}

22. ¿Hiciste trampa en los exámenes (e.g., usaste machetes o copiaste a un compañero)?

23. ¿Te echaron de clase por portarte mal (e.g., por conducta inapropiada o por copiar)?

24. ¿Te hiciste «la rata» cuando tus padres pensaban que estabas en clase?

25. ¿Perdiste clases intencionadamente durante varios días solo por diversión (e.g., no estabas enfermo o no había un motivo familiar)?

26. ¿Tuviste problemas en la escuela y tus padres recibieron un llamado 0 notificación sobre eso?

27. ¿Has faltado a clases 0 al trabajo haciendo creer que estabas enfermo?

\section{.49}

.68

.71

$\begin{array}{lllll}.88 & .52 & .21 & .08 & -.28^{*}\end{array}$ .86

.85

.87

.47

.75

.62

.53 63

.63

.78

.74

.82

.70

.83

.91

.80

$\begin{array}{llllll} & .75 & .34 & .05 & .04 & -.34^{*} \\ .44 & & & & & \\ .75 & & & & & \\ .55 & & & & & \\ & & & & \\ .47 & & & & \\ & & & & \\ .66 & & & & & \\ .54 & & & & & \\ \end{array}$




\begin{tabular}{|c|c|c|c|c|c|c|}
\hline Subescalas & $\lambda$ & CC & AVE & MSV & ASV & A \\
\hline Desviación general & & .74 & .27 & .10 & .07 & $-.29^{*}$ \\
\hline 28. ¿Has estado en la propiedad de alguien sabiendo que no debías estar ahí? & .53 & & & & & \\
\hline \multicolumn{7}{|l|}{ 29. ¿Has pinchado o sacado el aire de las llantas de un auto o bicicleta para } \\
\hline molestar a alguien? & .51 & & & & & \\
\hline 30. ¿Has hecho llamadas telefónicas molestas u obscenas? & .51 & & & & & \\
\hline 31. ¿Te has colado o te has ido de algún sitio sin pagar (e.g., cine, colectivo, bar o restaurant)? & .50 & & & & & \\
\hline 32. ¿Has usado dinero falso u otras cosas para sacar productos de las máquinas? & .49 & & & & & \\
\hline 33. ¿Has golpeado un auto estacionado solo para que sonase la alarma? & .54 & & & & & \\
\hline $\begin{array}{l}\text { 34. ¿Has pasado fuera toda la noche sin informar a tus padres sobre dónde estabas, } \\
\text { sabiendo que debías informarles? }\end{array}$ & .55 & & & & & \\
\hline 35. ¿Has superado el límite de velocidad solo para divertirte? & .51 & & & & & \\
\hline Robo & & .75 & .43 & .09 & .06 & $-.22^{*}$ \\
\hline \multicolumn{7}{|l|}{ 36. ¿Has robado, te has llevado o has intentado llevarte algo de tus familiares } \\
\hline (e.g., cosas personales o dinero)? & .57 & & & & & \\
\hline \multicolumn{7}{|l|}{ 37. ¿Has robado, te has llevado o has intentado llevarte algo de un negocio por valor de } \\
\hline 50 pesos o menos (e.g., periódicos, golosinas, dinero, etc.)? & .66 & & & & & \\
\hline \multicolumn{7}{|l|}{ 38. ¿Has robado, te has llevado o has intentado llevarte algo de un negocio por valor entre } \\
\hline 50 y 500 pesos (e.g., remeras, relojes, perfumes, videojuegos, CDs, zapatos, dinero, etc.)? & .59 & & & & & \\
\hline \multicolumn{7}{|l|}{ 39. ¿Has robado, te has llevado o has intentado llevarte algo «público» } \\
\hline (e.g., señales de tránsito, señales de construcción o bancos de una plaza)? & .79 & & & & & \\
\hline Agresión física & & .83 & .57 & .14 & .06 & $-.22^{\star}$ \\
\hline 40. ¿Has golpeado o amenazado con golpear a una persona? & .87 & & & & & \\
\hline 41. ¿Has golpeado o intentado golpear a tus padres? & .42 & & & & & \\
\hline 42. ¿Has golpeado o amenazado con golpear a estudiantes, compañeros 0 a otras personas? & .90 & & & & & \\
\hline 43. ¿Has dado una paliza tan fuerte a alguien que requirió atención médica? & .74 & & & & & \\
\hline
\end{tabular}

Nota: $\lambda=$ Cargas factoriales estandarizadas; $\mathrm{CC}=$ Confiabilidad compuesta; AVE = Variable media extraída; MSV = Máxima varianza compartida al cuadrado; ASV = Varianza media al cuadrado compartida; A = Variable Autocontrol asociada con las dimensiones de la NDS. $* p<.01$

\section{Análisis de consistencia interna}

Para analizar la consistencia interna de la escala se utilizó el índice de confiabilidad compuesta. Como resultado de este análisis se obtuvo que los índices de confiabilidad compuesta fueron adecuados para las siete subescalas, variando entre .74 (Desviación general) a .92 (Consumo de drogas) (ver Tabla 2). Validez convergente, discriminante y concurrente.

Los resultados del análisis de validez convergente mediante la AVE indicaron que solo cuatro de las siete subescalas de la EDN cumplieron con el criterio AVE $>.40$, lo que indica que solo estas dimensiones poseen adecuadas propiedades de validez convergente. De las restantes subescalas, la dimensión desviación general presentó el valor de AVE más bajo. Con respecto al análisis de validez discriminante, los resultados arrojaron que tanto la MSV como la ASV fueron menores a la AVE para las siete subescalas, confirmándose así la presencia de validez discriminante en términos estadísticos en dichas subescalas (ver Tabla 2). Además, con respecto a la validez concurrente, la Tabla 2 muestra correlaciones negativas, bajas, moderadas y significativas $(p<.01)$ entre las dimensiones de la EDN y la variable autocontrol. 
Por último, se evalúo la invarianza de medición del modelo de medida con mejor ajuste estadístico y los resultados a nivel general no sustentaron la invarianza factorial entre los grupos conformados según el sexo de los participantes (ver Tabla 3).

Tabla 3

Índices de bondad de ajuste y comparación de los modelos para evaluar la invariancia de medidas

\begin{tabular}{lcccccccc}
\hline \multicolumn{1}{c}{ Modelo } & $\chi^{2}$ & $d f$ & TLI & CFI & RMSEA [95\% IC] & $\Delta$ TLI & $\Delta$ CFI & $\Delta$ RMSEA \\
\hline $\begin{array}{l}\text { Invarianza } \\
\text { configural }\end{array}$ & 2913.368 & 1681 & .895 & .902 & $.043[.040, .046]$ & - & - & - \\
$\begin{array}{l}\text { Invarianza } \\
\text { métrica }\end{array}$ & 2727.628 & 1711 & .915 & .919 & $.039[.036, .041]$ & .02 & .017 & .004 \\
$\begin{array}{l}\text { Invarianza } \\
\text { escalar }\end{array}$ & 3137.483 & 1883 & .905 & .901 & $.041[.038, .043]$ & .01 & .018 & .002 \\
\hline
\end{tabular}

Nota: $\chi^{2}$ = Chi-Cuadrado; $d f$ = Grados de libertad; TLI = Índice de Tucker-Lewis; CFI = Índice de ajuste comparativo; RMSEA = Raíz cuadrada media del error de aproximación.

\section{Discusión}

Las conductas vinculadas con la transgresión de la norma, ya sea que impliquen una sanción legal o social, continúan siendo el centro de investigación en diversos campos disciplinarios debido a sus graves consecuencias (Moffitt, 1993). En el contexto argentino, las estadísticas a nivel nacional y provincial reportan que en jóvenes es frecuente la prevalencia de comportamientos en contra de la norma (Ministerio de Justicia y Derechos Humanos, 2017a, 2017b). Por este motivo, el objetivo de nuestra investigación fue determinar si la EDN es un instrumento que permite valorar el comportamiento antisocial en jóvenes de forma confiable y valida. Con este propósito, se evalúo el ajuste estadístico, la confiabilidad y validez de las siete dimensiones de la escala.

Con anterioridad a los análisis para evaluar el ajuste estadístico del modelo de medida, se obtuvieron datos sobre la prevalencia de las conductas antisociales. Como resultado se encontró que más del 50\% de los jóvenes que participaron del estudio, al menos una vez cometieron algún tipo de comportamiento antisocial. Estos resultados concuerdan con los reportados en estudios anteriores que reafirman la presencia de estas conductas en la franja etaria que va desde los 18 a 30 años (Vazsonyi et al., 2004). Además, los datos obtenidos en nuestra muestra permiten confirmar que las conductas antinormativas no se limitan solo a poblaciones en conflicto con la ley penal, sino que también se presentan en población general (Vazsonyi et al., 2001).

Con respecto a los análisis estadísticos sobre el modelo de medida original, se encontraron valores aceptables para el índice de confiabilidad. Sin embargo, el análisis factorial confirmatorio indicó que la estructura interna de la escala no era adecuada. En nuestro caso, al evaluar el modelo de medida original, se produjo la premisa número tres analizada por Stanley y Edwards (2016). Esta premisa refiere a la situación en que la evaluación de la confiabilidad y el ajuste del modelo parecen proporcionar pruebas contradictorias sobre el instrumento de medición. En este caso, los autores siguiendo las consideraciones de Schmitt (1996) recomiendan evaluar ambas fuentes de información para tomar decisiones, ya que ninguna de ellas de forma independiente es suficiente para demostrar que un modelo de medición es compatible con los datos empíricos. Por este motivo, pese a los adecuados niveles de confiabilidad, se decidió continuar con el AFC y se logró arribar a una estructura factorial que a nivel general presenta 
armonía entre el índice de confiabilidad y el ajuste del modelo de medida.

Cabe mencionar que en su gran mayoría los índices de ajuste estadístico del modelo presentaron valores adecuados excepto el WRMR. Este índice se considera como un índice de ajuste experimental (Muthén y Muthén, 1998-2012) y se encuentra condicionado por las categorías de respuesta de la escala, por el tamaño de la muestra y por el incremento de los niveles de anormalidad en la distribución de los datos (DiStefano, Liu, Jiang, \& Shi, 2018). Asimismo, como lo menciona DiStefano et al. (2018), en la investigación de carácter empírica es necesario interpretar de manera holística los resultados del ajuste del modelo de medida, ya que no se debe confiar en un solo índice como evidencia de un buen o mal ajuste del modelo. Por lo cual, en aquellos casos en que indicadores como el WRMR no se ajusten a los criterios estadísticos propuestos para su interpretación, esto no es suficiente evidencia para descartar un modelo de medida (DiStefano et al., 2018). Además, si este valor se aproxima al criterio estadístico de interpretación y los indicadores como el CFI, TLI o RMSEA que poseen una alta sensibilidad para identificar una especificación inadecuada y no dependen del tamaño de la muestra son adecuados, es viable aceptar el modelo de medida (Hu \& Bentler, 1998).

Con respecto a la consistencia interna del modelo de medida final, las siete dimensiones obtuvieron valores de confiabilidad compuesta adecuados. Estos resultados se encuentran en línea con estudios previos realizados en contextos culturales diferentes (Vazsonyi, et al., 2004), en población general (Coffey, Cox, J, \& Kopkin, 2018) y en muestras compuestas por adolescentes (Torrente \& Vazsonyi, 2012). Además, considerando los criterios estadísticos para tener en cuenta un adecuado nivel de confiabilidad en los instrumentos de medición psicológica (Nunnally, 1978), se puede considerar a la EDN un instrumento adecuado para evaluar la conducta antisocial en el área de investigación.
Con relación a la validez de la prueba, el análisis de validez convergente indicó que las dimensiones Consumo de alcohol, Consumo de droga, Robo y Agresión física presentaron adecuados valores en el índice AVE (Etchezahar et al., 2014). Sin embargo, las dimensiones Vandalismo, Mala conducta escolar y Desviación general no cumplieron con este criterio. Esto puede deberse a que la AVE es sensible a modificaciones en función del número de ítems y de la homogeneidad de las cargas factoriales, las cuales deberían ser en promedio e» .70 para obtener un valor apropiado.

Por su parte, los índices MSV y ASV para evaluar la validez discriminante fueron adecuados, lo que confirma que los ítems definen solo la dimensión a la que pertenecen (Hair et al., 2010). Si comparamos estos resultados con estudios que evaluaron las dimensiones de la escala y su correlación con diferentes constructos psicológicos, encontramos que nuestros resultados están en consonancia, por ejemplo, con los obtenidos en el estudio realizado por Torrente y Vazsonyi (2012). Estos autores, al correlacionar las dimensiones Consumo de alcohol, Consumo de droga, Vandalismo y Robo entre sí, encontraron correlaciones positivas y moderadas. Además, cuando correlacionaron estas cuatro dimensiones con Amabilidad y Responsabilidad encontraron correlaciones bajas y negativas.

Con respecto a los resultados del estudio de validez criterio se encontraron correlaciones negativas, moderadas y estadísticamente significativas entre las siete dimensiones de la EDN y el autocontrol. Estos resultados concuerdan con investigaciones previas realizadas en población general (adolescentes, jóvenes y adultos) que concluyeron que a menores niveles en la variable autocontrol, mayor es la presencia de conductas antisociales (Cihan \& Tittle, 2019; Ridder, Lensvelt-Mulders, Finkenauer, Stok, \& Baumeister, 2018; Vazsonyi \& Jiskrova, 2018). Además, nuestros resultados concuerdan con la direccionalidad y el grado de asociación que manifiesta la conducta antisocial y el autocontrol en estudios previos (Baron, 
2003; Bunch, Iratzoqui, \& Watts, 2018; Chui \& Chan, 2013; Tillyer et al., 2018; Walters et al., 2018). Por ejemplo, en el estudio de Vazsonyi et al. (2004), quienes compararon una muestra de estudiantes universitarios de origen japonés y una de origen estadounidense, encontraron que en ambas muestras los valores de las correlaciones fueron bajas y positivas entre las siete dimensiones de la EDN y el bajo autocontrol, siendo el valor menor de correlación .16 (Agresión física) y el mayor valor de correlación .27 (Consumo de alcohol).

Por último, los resultados del análisis de invarianza factorial no permitieron comprobar empíricamente la equivalencia del modelo de medida considerando el sexo de los participantes de la muestra. Lo cual implica que el grado de generalización de las puntuaciones y las interpretaciones que de ellas podrían derivarse, no pueden ser utilizados de manera equivalente en hombres y mujeres para referirse al nivel en el rasgo medido (Chen, 2007). Asimismo, cabe mencionar que el análisis de equivalencia puede estar siendo afectado por las características específicas de la muestra, tal como lo menciona la literatura, los hombres presentan puntuaciones significativamente más altas que las mujeres en conductas como el Consumo de alcohol (Chaiyasong et al., 2018), Consumo de drogas (Cuttler, Mischley, \& Sexton, 2016), Vandalismo (Nordmarker et al., 2016), Agresión física (Card et al., 2008), Desviación general (Kobayashi, Sharp, \& Grasmick, 2008) y Robo (Hoertel et al. 2012).

\section{Limitaciones y sugerencias de futuras investigaciones}

Como en la mayoría de los trabajos de investigación, este no está exento de limitaciones. Entre las limitaciones se puede mencionar que los resultados no permitieron comprobar la validez convergente en tres de las siete dimensiones que componen la escala, la cual puede estar siendo afectada por la cantidad y el peso factorial de los ítems que componen cada factor. Por este motivo, aunque utilizar criterios estadísticos como la AVE, la MSV y la ASV (para evaluar la validez convergente y discriminante) sea adecuado, se recomienda que en futuros estudios se obtengan indicadores de validez asociados a otras variables mediante fuentes de comparación externa (American Educational Research Association, American Psychological Association, \& National Council on Measurement in Education, 2014). Además, no se puede asegurar que el instrumento de medición funciona de igual manera para hombres y mujeres, por lo que se sugiere que en futuras investigaciones para evaluar la equivalencia de medida se considere el tiempo de aplicación (e.g., en estudios longitudinales), los medios de administración (e.g., versión lápiz y papel, y versión online), las diferencias culturales o la edad de los participantes (Meade, Michels, \& Lautenschlager, 2007; Stein, Lee, \& Jones, 2006; Widaman, Ferrer, \& Conger, 2010). Además, se recomienda considerar los aportes de la teoría de respuesta al ítem, ya que se podría evaluar mediante el análisis diferencial de los ítems, por ejemplo, si tanto hombres como mujeres con igual nivel en el rasgo latente poseen la misma probabilidad de obtener puntuaciones observadas semejantes en la escala.

Si bien el estudio presentó limitaciones, cabe resaltar que los análisis aquí realizados resultan un avance y aporte al estudio de la conducta antisocial en una muestra de jóvenes argentinos. Además, la escala cumplió con la mayoría de los criterios de validez y confiabilidad, por lo que podría ser utilizada con fines de investigación (Nunnally, 1978). También cabe resaltar que la EDN es un instrumento que no se basa en términos legales o estrictamente conductuales, lo que permite evaluar actos antisociales más allá de las consideraciones específicas que puede disponer una determinada cultura o sociedad (Gottfredson \& Hirschi, 1990).

\section{Conflicto de intereses}

Todos los autores del presente manuscrito manifestamos que no existe conflicto de intereses. 


\section{Responsabilidad ética}

En el presente estudio de investigación no se realizaron experimentos en seres humanos ni en animales. La participación de los jóvenes en la investigación se realizó considerando las recomendaciones de la legislación de la República Argentina para la protección de los datos personales (Ley $\mathrm{N}^{\circ} 25.326$ ) y de la Asociación Estadounidense de Psicología (APA, 2016), por lo que se les informó a los participantes la confidencialidad de sus respuestas, el carácter voluntario de su participación y su derecho a retirarse en cualquier instancia del estudio sin que esto conlleve algún perjuicio para su persona. Con el fin de resguardar la confidencialidad de los datos personales, en el presente estudio no se brinda información de los participantes que permita identificarlos.

\section{Contribución de autoría}

SJG: concepción, búsqueda bibliográfica, diseño del estudio, recolección de datos, revisión y corrección de las versiones preliminares y final del manuscrito.

KA: concepción, diseño del estudio, recolección de datos, análisis estadístico y revisión final.

MC: concepción, diseño del estudio, recolección de datos, análisis estadístico y revisión final.

FBG: análisis estadístico, interpretación de los resultados, contribuyó con la discusión, revisiones preliminares y final de manuscrito.

AEA: análisis estadístico, interpretación de los resultados, contribuyó con la discusión y la revisión final de manuscrito.

VEM: participación y asesoramiento del proceso de investigación (en diferentes instancias), corrección de la primera versión del manuscrito y revisión final de manuscrito.

\section{Referencias}

American Educational Research Association, American Psychological Association, \& National Council on Measurement in Education. (2014). Standards for Educational and Psychological Testing. Washington, DC: American Educational Research Association.

American Psychological Association. (2016). Publication Manual of the American Psychological Association (6 $6^{\text {ta }}$ ed.). Washington, DC: American Psychological Association.

Baron, S. W. (2003). Self-Control, Social Consequences, and Criminal Behavior: Street Youth and the General Theory of Crime. Journal of Research in Crime and Delinquency, 40(4), 403-425. doi: 10.1177/0022 427803256071

Bobbio, A., Arbach, K., \& Vazsonyi, A. T. (2019). Selfcontrol and Deviance: A Test of the General Theory of Crime in Argentina. Victims \& Offenders, 14(1), 119-142. doi: 10.1080/15564886.2018.1552222

Brown, T. A. (2006). Confirmatory factor analysis for applied research: New York: Guilford.

Byrne, B. (2008). Testing for multigroup equivalence of a measuring instrument: Awalk through the process. Psicothema, 20(4), 872-882.

Bunch, J. M., Iratzoqui, A., \& Watts, S. J. (2018). Child abuse, self-control, and delinquency: A general strain perspective. Journal of Criminal Justice, 56, 20-28. doi: 10.1016/j.jcrimjus.2017.09.009

Butchart, A., \& Mikton, C. (2014). Global status report on violence prevention, 2014. Technical Report. World Health Organization.

Card, N. A., Stucky, B. D., Sawalani, G. M., \& Little, T. D. (2008). Direct and indirect aggression during childhood and adolescence: A meta analytic review of gender differences, intercorrelations, and relations to maladjustment. Child development, 79(5), 11851229. doi: 10.1111/j.1467-8624.2008.01184.x

Chaiyasong, S., Huckle, T., Mackintosh, A. M., Meier, P., Parry, C. D., Callinan, S., ... Casswell, S. (2018). Drinking patterns vary by gender, age and country level income: Cross country analysis of the International Alcohol Control Study. Drug and Alcohol Review, 37, 53-62. doi: 10.1111/dar.12820 
Chen, F. F. (2007). Sensitivity of goodness of fit indexes to lack of measurement invariance. Structural Equation Modeling, 14(3), 464-504. doi: 10.1080/ 10705510701301834

Cheung, G. W., \& Rensvold, R. B. (2002). Evaluating Goodness-of-Fit Indexes for Testing Measurement Invariance. Structural Equation Modeling, 9(2), 233255. doi: 10.1207/S15328007SEM0902_5

Chui, W. H., \& Chan, H. C. (2013). The Gendered Analysis of Self-Control on Theft and Violent Delinquency: An Examination of Hong Kong Adolescent Population. Crime \& Delinquency, 62(12), 1648-1677. doi: 10.1177/00111287124 70992

Cihan, A., \& Tittle, C. R. (2019). Self-Control, Sanction Threats, Temptation, and Crime: Examining Contingencies of Self-Control in a Cross-National Context. Crime \& Delinquency, 65(4), 555-580. doi: 10.1177/0011128718824939

Coffey, C. A., Cox, J., \& Kopkin, M. R. (2018). Examining the Relationships Between the Triarchic Psychopathy Constructs and Behavioral Deviance in a Community Sample. Journal of Personality Disorders, 32(1), 57-69. doi: 10.1521/pedi_2017_3 1_288

Cohen, J. (1988). Statistical Power Analysis for the Behavioral Sciences. ( $2^{\mathrm{da}}$ ed.). Hillsdate, NJ: Lawrence Erlbaum Associates.

Cuttler, C., Mischley, L. K., \& Sexton, M. (2016). Sex Differences in Cannabis Use and Effects: A CrossSectional Survey of Cannabis Users. Cannabis and Cannabinoid Research, 1(1), 166-175. doi: 10.1089/ can.2016.0010

Dimitrov, D. M. (2010). Testing for factorial invariance in the context of construct validation. Measurement and Evaluation in Counseling and Development, 43(2), 121-149.

DiStefano, C., Liu, J., Jiang, N., \& Shi, D. (2018). Examination of the weighted Root Mean Square Residual: Evidence for Trustworthiness? Structural Equation Modeling: A multidisciplinary Journal, 25(3), 453-466. doi: 10.1080/10705511.2017.1 390394
Eitle, D., Niedrist, F., \& Eitle, T. M. (2014). Gender, Race, and Delinquent Behavior: An Extension of Power-Control Theory to American Indian Adolescents. Deviant Behavior, 35(12), 1023-1042. doi: 10.1080/01639625.2014.903756

Etchezahar, E., Prado-Gascó, V., Jaume, L., \& Brussino, S. (2014). Argentinian validation of the Social Dominance Orientation Scale. Revista Latinoamericana de Psicología, 46(1), 35-43.

Gaik, L. P., Abdullah, M. C., Elias, H., \& Uli, J. (2010). Development of Antisocial Behaviour. ProcediaSocial and Behavioral Sciences, 7, 383-388. doi: 10.1016/j.sbspro.2010.10.052

Garrido, S. J., Cupani, M., \& Arbach, K. (2017). Application of the rating scale model to examine the psychometric properties of the self-control scale. Psykhe, 26(2), 1-12. doi:10.7764/psykhe.26.2.980

Garrido, S. J., Morán, V., Azpilicueta, A. E., Cortez, F., Arbach, K., \& Cupani, M. (2018). Análisis de modelos rivales unidimensionales y bidimensionales de la escala breve de autocontrol en estudiantes universitarios argentinos. Psicodebate. Psicología, Cultura y Sociedad, 18(2), 26-37.

George, D., \& Mallery, P. (2011). SPSS for Windows Step by Step: A Simple Guide and Reference 18.0 Update (11 ${ }^{\text {va }}$ ed.). Boston, MA: Allyn y Bacon.

Gottfredson, M. R., \& Hirschi, T. (1990). A general theory of crime. Stanford: Stanford University Press.

Gunzler, D. D., \& Morris, N. (2015). A tutorial on structural equation modeling for analysis of overlapping symptoms in co occurring conditions using MPlus. Statistics Inmedicine, 34(24), 32463280. doi: 10.1002/sim.6541

Hair, J. F., Black, W. C., Babin, B. J., \& Anderson, R. E. (2010). Multivariate Data Analysis ( $7^{\mathrm{ma}}$ ed.). Englewood Cliffs: Prentice Hall.

Hoertel, N., Dubertret, C., Schuster, J. P., \& Le Strat, Y. (2012). Sex Differences in Shoplifting: Results From a National Sample. The Journal of Nervous and Mental Disease, 200(8), 728-733. doi: 10.1097/nm d.0b013e3182613fbb 
Hu, L., \& Bentler, P. M. (1998). Fit indices in covariance structure modeling: Sensitivity to underparameterized model misspecification. Psychological Methods, 3, 424-453. doi: 10.1037/1082-989X.3.4.424

Kobayashi, E., Sharp, S. F., \& Grasmick, H. G. (2008). Gender and Deviance: A Comparison of College Students in Japan and the United States. Deviant Behavior, 29(5), 413-439. doi: 10.1080/01900 690701598010

Ley $\mathrm{N}^{\circ}$ 25.326. Colocar título de la publicación en que aparece oficialmente la ley. Argentina, 2 de noviembre de 2000.

Meade, A. W., Michels, L. C., \& Lautenschlager, G. J. (2007). Are Internet and Paper-and-Pencil Personality Tests Truly Comparable?: An experimental Design Measurement Invariance Study. Organizational Research Methods, 10(2), 322-345. doi: 10.1177/10 94428106289393

Ministerio de Justicia y Derechos Humanos. (2017a). Informe Anual República Argentina Sistema Nacional de Estadística sobre Ejecución de la Pena 2017. Recuperado de: https://www.argentina.gob.ar/sites/ default/files/informe_sneep_argentina_2017.pdf

Ministerio de Justicia y Derechos Humanos. (2017b). Informe Anual Córdoba Sistema Nacional de Estadística sobre Ejecución de la Pena 2017. Recuperado de: https://www.argentina.gob.ar/sites/ default/files/sneepcordoba2017.pdf

Moffitt, T. E. (1993). Adolescence-Limited and LifeCourse-Persistent Antisocial Behavior: A Developmental Taxonomy. Psychological Review, 100(4), 674-701. doi: 10.1037/0033-295X.10 0.4 .674

Montero, I., \& León, O. G. (2007). A guide for naming research studies in Psychology. International Journal of Clinical and Health Psychology, 7(3), 847-862.

Muthén, L. K., \& Muthén, B. O. (2011). Mplus user's guide ( $5^{\text {ta }}$ ed.). Los Angeles, CA: Muthén \& Muthén.

Muthén, L. K., \& Muthén, B. O. (1998-2012). Mplus user's guide ( $7^{\mathrm{ma}}$ ed.). Los Angeles, CA: Muthén \& Muthén.
Nordmarker, A., Hjärthag, F., Perrin-Wallqvist, R., \& Archer, T. (2016). The roles of gender and personality factors in vandalism and scrawl-graffiti among Swedish adolescents. PsyCh Journal, 5(3), 180-190. doi: 10.1002/pchj.133

Nunnally, J. C. (1978). Psychometric theory (2da ed.). New York: McGraw-Hill.

Ridder, D. T. D. de, Lensvelt-Mulders, G., Finkenauer, C., Stok, F. M., \& Baumeister, R. F. (2012). Taking Stock of Self-Control: A Meta-Analysis of How Trait SelfControl Relates to a Wide Range of Behaviors. Personality and Social Psychology Review, 16(1), 76-99. doi: 10.1177/1088868311418749

Schmitt, N. (1996). Uses and abuses of coefficient alpha. Psychological Assessment, 8, 350-353.

Stanley, L. M., \& Edwards, M. C. (2016). Reliability and Model Fit. Educational and Psychological Measurement, 76(6), 976-985. doi: 10.1177/001 3164416638900

Stein, J. A., Lee, J. W., \& Jones, P. S. (2006). Assessing Cross-Cultural Differences Through Use of MultipleGroup Invariance Analyses. Journal of Personality Assessment, 87(3), 249-258. doi: 10.1207/s15327 752jpa8703_05

Tangney, J. P., Baumeister, R. F., \& Boone, A. L. (2004). High Self Control Predicts Good Adjustment, Less Pathology, Better Grades, and Interpersonal Success. Journal of Personality, 72, 271-324. doi: 10.1111/ j.0022-3506.2004.00263.x

Tillyer, M. S., Wilcox, P., \& Fissel, E. R. (2018). Violence in Schools: Repeat Victimization, Low Self-Control, and the Mitigating Influence of School Efficacy. Journal of Quantitative Criminology, 34(2), 609632. doi: 10.1007/s10940-017-9347-8

Thornberry, T. P., \& Krohn, M. D. (2000). The SelfReport Method for Measuring Delinquency and Crime. Criminal Justice, 4(1), 33-83.

Torrente, G., \& Vazsonyi, A. T. (2012). Personality, Parenting and Deviance Among Spanish Adolescents. Anales de Psicología, 28(3), 654-664. doi: 10.6018/ analesps.28.3.155951 
Vazsonyi, A. T., \& Jiskrova, G. K. (2018). On the development of self-control and deviance from preschool to middle adolescence. Journal of Criminal Justice, 56, 60-69. doi: 10.1016/j.jcri mjus.2017.08.005

Vazsonyi, A. T., Pickering, L. E., Junger, M., \& Hessing, D. (2001). An Empirical Test of a General Theory of Crime: A four-Nation Comparative Study of SelfControl and the Prediction of Deviance. Journal of Research in Crime and Delinquency, 38(2), 91-131. doi: $10.1177 / 0022427801038002001$

Vazsonyi, A. T., Wittekind, J. E. C., Belliston, L. M., \& Loh, T. D. van (2004). Extending the General Theory of Crime to «The East»: Low Self-Control in Japanese Late Adolescents. Journal of Quantitative Criminology, 20(3), 189-216. doi: 10.1023/B:JOQ C.0000037731.28786.e3

Valentini, F., \& Damásio, B. F. (2016). Variância Média Extraída e Confiabilidade Composta: Indicadores de Precisão. Psicologia: Teoria e Pesquisa, 32(2), e322225. doi: 10.1590/0102-3772e322225

Walters, K. J., Simons, J. S., \& Simons, R. M. (2018). Self-control demands and alcohol-related problems: Within- and between-person associations. Psychology of Addictive Behaviors, 32(6), 573-582. doi. 10.1 037/adb0000387
Webb, V. J., Katz, C. M., \& Decker, S. H. (2006). Assessing the Validity of Self-Reports by Gang Members: Results From the Arrestee Drug Abuse Monitoring Program. Crime \& Delinquency, 52(2), 232-252. doi: $10.1177 / 0011128705277972$

Widaman, K. F., Ferrer, E., \& Conger, R. D. (2010). Factorial invariance Within Longitudinal Structural Equation Models: Measuring the Same Construct Across Time. Child Development Perspectives, 4(1), 10-18. doi: 10.1111/j.1750-8606.2009.00110.x

Whittaker, T. A. (2012). Using the Modification Index and Standardized Expected Parameter Change for Model Modification. The Journal of Experimental Education, 80 (1), 26-44. doi: 10.1080/0022097 3.2010 .531299

World Health Organization. (2015). Preventing youth violence: An overview of the evidence. Geneva, Switzerland: World Health Organization Press.

Yu, C. Y., \& Muthén, B. O. (2002). Evaluation of Model Fit Indices for Latent Variable Models with Categorical and Continuous Outcomes. Trabajo presentado en la conferencia de la Asociación Americana de Investigación Educativa, New Orleans.

\section{Sebastián Jesús Garrido}

Facultad de Psicología, Universidad Nacional de Córdoba, Argentina

Instituto de Investigaciones Psicológicas, IIPSI, Unidad Ejecutora CONICET, Córdoba, Argentina

Licenciado en Psicología, Becario doctoral CONICET. Su línea de investigación y publicaciones se centran en los factores de riesgo del comportamiento desadaptativo (internalizado y externalizado), la personalidad y la construcción y adaptación de instrumentos de medición en el campo de la Psicología.

ORCID: 0000-0001-6603-7285

Autor corresponsal: sebajgarrido@gmail.com

\section{Karin Arbach}

Facultad de Psicología, Universidad Nacional de Córdoba, Argentina Instituto de Investigaciones Psicológicas, IIPSI, Unidad Ejecutora CONICET, Córdoba, Argentina.

Doctora en Psicología, profesora titular de la Facultad de Psicología (Universidad Nacional de Córdoba), investigadora del CONICET. Su línea de investigación y publicaciones se centran en la evaluación y gestión del riesgo de violencia.

ORCID: 0000-0003-1764-0090

k_arbach@hotmail.com 


\section{Marcos Cupani}

Facultad de Psicología, Universidad Nacional de Córdoba, Argentina

Universidad Nacional de Córdoba, Facultad de Psicología, Córdoba, Argentina

Instituto de Investigaciones Psicológicas, IIPSI, Unidad Ejecutora CONICET, Córdoba, Argentina

Droctor: en Psicología, profesor titular de la Facultad de Psicología (Universidad Nacional de Córdoba) e investigador del CONICET. Su línea de investigación y publicaciones se centran en identificar diferencias individuales que permiten predecir el rendimiento académico y la construcción y adaptación de pruebas psicológicas.

ORCID: 0000-0003-2132-5552

marcoscup@gmail.com

\section{Fernanda Belén Ghío}

Facultad de Psicología, Universidad Nacional de Córdoba, Argentina

Universidad Nacional de Córdoba, Facultad de Psicología, Córdoba, Argentina

Instituto de Investigaciones Psicológicas, IIPSI, Unidad Ejecutora CONICET, Córdoba, Argentina

Licenciada en Psicología, Becaria doctoral FONCYT (Universidad Nacional de Córdoba). Su línea de investigación y publicaciones se centran en el rendimiento académico, la personalidad, la inteligencia y la construcción de pruebas psicométricas.

ORCID: 0000-0002-4223-2470

fernandabghio@gmail.com

\section{Ana Estefanía Azpilicueta}

Facultad de Psicología, Universidad Nacional de Córdoba, Argentina

Universidad Nacional de Córdoba, Facultad de Psicología, Córdoba, Argentina

Instituto de Investigaciones Psicológicas, IIPSI, Unidad Ejecutora CONICET, Córdoba, Argentina

Doctora en Psicología, su línea de investigación y publicaciones se centran en la evaluación longitudinal, las creencias de autoeficacia, los factores contextuales, las variables cognitivas involucradas en el decisión de carrera y la construcción de pruebas psicológicas.

ORCID: 0000-0002-6221-3162

estefaniaazpilicueta@gmail.com

\section{Valeria Estefanía Moran}

Facultad de Psicología, Universidad Nacional de Córdoba, Argentina

Universidad Nacional de Córdoba, Facultad de Psicología, Córdoba, Argentina

Instituto de Investigaciones Psicológicas, IIPSI, Unidad Ejecutora CONICET, Córdoba, Argentina

Doctora en Psicología, cuenta con investigaciones en comportamiento interpersonal en estudiantes universitarios y ansiedad social. Su línea de formación se focaliza en la construcción y adaptación de instrumentos de medición.

ORCID: 0000-0003-3628-1636

moranvaleria@gmail.com 